\title{
Characteristic Mode Analysis applied to antennas
}

\author{
Marcelo B. Perotoni*1@, Felipe A. A. da Silva ${ }^{1}$, Leandro A. da Silva ${ }^{1}$ \\ ${ }^{1}$ Universidade Federal do ABC, Santo Andre, SP, Brasil
}

Received on April 01, 2020. Accepted on May 23, 2020.

\begin{abstract}
This paper introduces the Characteristic Mode Analysis in the computation of electromagnetic resonant modes in conducting bodies. Canonical cases (thin wire and a rectangular plate) are analyzed, along with real-world complex structures, a mobile phone chassis and an unmanned aerial vehicle. Given the analogy to other areas, such as modal analysis in Differential Equations, this method can be another tool when teaching Electromagnetic Radiation and antenna related topics.
\end{abstract}

Keywords: Antennas, characteristic mode analysis, computational electromagnetics.

\section{Introduction and physical meaning of the modes}

The Characteristic Mode Analysis (CMA) generates a set of orthogonal real currents on a conducting body with arbitrary geometry. Similar to Fourier Series Expansion, where a sum of orthogonal harmonic functions (sines and cosines) describes arbitrary waveforms, CMA expresses the existing current patterns in a body using its natural modes of resonance. Those modes are, therefore, dependent on the body geometry and independent of the excitation. The excitation (geometry and location) is relevant later on, when determining which particular mode(s) will be present.

The theory behind CMA was first developed by Garbacz [1], in 1971. Later, Harrington improved on the existing applications and proposed a simpler derivation [2]. The theory fell into disuse later [3], appearing again in the technical literature only during the first decades of 2000, taking advantage of tools offered by commercial 3D field solvers that had this option available, especially the ones which were based on the Method of Moments (MoM).

The CMA is based on the following weighted eigenvalue equation [3]:

$$
X\left(\overrightarrow{J_{n}}\right)=\lambda_{n} R\left(\overrightarrow{J_{n}}\right)
$$

where $\lambda_{n}$ are the eigenvalues, $J_{n}$ the eigencurrents. The complex impedance $Z$ is defined as

$$
Z=R+j X
$$

Then the total current on the structure can be described as a weighted sum of the orthogonal components [4]:

$$
\vec{J}=\sum_{n} \alpha_{n} J_{n}=\sum_{n} \frac{V_{n}^{i} \vec{J}_{n}}{1+j \lambda_{n}}
$$

\footnotetext{
*Correspondence email address: marcelo.perotoni@ufabc.edu.br
}

where $\alpha_{n}$ is the parameter called modal weighting coefficient and $V_{n}^{i}$ is the modal excitation coefficient, defined as:

$$
V_{n}^{i}=\oint_{S} \overrightarrow{J_{n}} \cdot \vec{E}^{i} d S
$$

where $E^{i}$ is the impressed source. In terms of radiation (conducting body operating as an antenna), each mode radiates independently, following the orthogonality. The eigenvalue $\lambda_{n}$ gives insight into the nature of the mode if its absolute value is zero it means the mode is radiating. Regarding the signal, negative $\left(\lambda_{n}<0\right)$ modes are storing electric energy and positive $\left(\lambda_{n}>0\right)$, magnetic.

Among the several parameters describing the CMA, the characteristic angle $\beta_{n}$ can be written as:

$$
\beta_{n}=180^{\circ}-\operatorname{atan}\left(\lambda_{n}\right)
$$

So for a given mode to be resonant its characteristic angle should be $180^{\circ}$, which is equivalent to its eigenmode $\lambda$ being zero.

Other parameter concerning the CMA, derived from the eigenmode, is the Mode Significance (MS), defined for the $\mathrm{n}$-th mode as:

$$
M S_{n}=\left|\frac{1}{1+j \lambda_{n}}\right|
$$

MS has a range from 0 to 1 - the latter meaning the radiation case.

These equations are numerically solved using a discretized scheme. One appropriate numerical approach is the Method of Moments (MoM), which creates a superficial (i.e with zero thickness, sheet-like) mesh on the metallic areas, therefore saving up computation effort when compared with volumetric meshes (e.g. FEM Finite Element Method or FDTD - Finite Difference Time Domain). Besides that, for the MoM the unknown 
variable is the current density on the structure, also the variable of CMA. The simulations here performed used the CST Microwave Studio Integral Equation Solver, based on the Method of Moments, a commercial package. Three case studies are covered to show the potential and physical meaning of the modes found by the CMA.

Electromagnetic radiation is traditionally seen by students as a difficult topic, heavily based on Mathematics with few physical analogies. Given the similarity to other resonant cases previously seen in classroom by STEM courses, such as a guitar string (1D) [5], drum/membrane modes (2D), as solutions to Differential Equations (Modal Analysis) [6], the use of CMA to introduce a physical insight into radiation and antenna cases seems to be attractive for undergraduate programs.

\section{Case study I: strip dipole}

For the first case, a metallic strip (1 meter length and $1 \mathrm{~cm}$ width, zero thickness) is simulated. According to the theory, the resonant half-wavelength would occur at approximately 0.48 times the free-space wavelength (the difference between the 0.5 and 0.48 is ascribed to the velocity of the wave on the metal [7]), which is equivalent to $143 \mathrm{MHz}$ for a 1-meter long thin wire. Figure 1 shows both the three first eigenmode values and the characteristic angles simulated. Taking into consideration frequencies where the eigenmodes are zero (radiating zone), smaller frequencies store electric energy; larger frequencies store magnetic energy. This is seen in wire antennas - electrically short antennas are capacitive and long antennas inductive, from the viewpoint of input impedance. Table 1 shows that the three first modes correspond to the known resonances $-0.5 \lambda, \lambda$ and $1.5 \lambda$.

The three first modes are constrained by the boundary conditions on the wire antenna, namely minimum current/maximum electric field on the strip edges (open circuit). Figure 2 shows the normalized current surface for the three first eigenmodes, with arrows pointing the nulls. It can be seen, on the interrupted lines, the similarity with the normal mechanical (oscillatory) modes seen on a uniform vibrating string. The respective farfield for the three different modes is shown in Figure 3 - it can be seen that there is a direct relation between the current surface pattern and the farfield pattern, a Fourier transform operator [7].

Regarding a real-world antenna, a proper excitation should be placed somewhere on the structure. Usually, wire antennas have the connection right on the middle point. From the viewpoint of excitation, two methods are

Table 1: First three modes

\begin{tabular}{lcc}
\hline Mode & Fraction of wavelength & Frequency $[\mathrm{MHz}]$ \\
\hline 1 & .48 & 143 \\
2 & .97 & 291 \\
3 & 1.47 & 440 \\
\hline
\end{tabular}

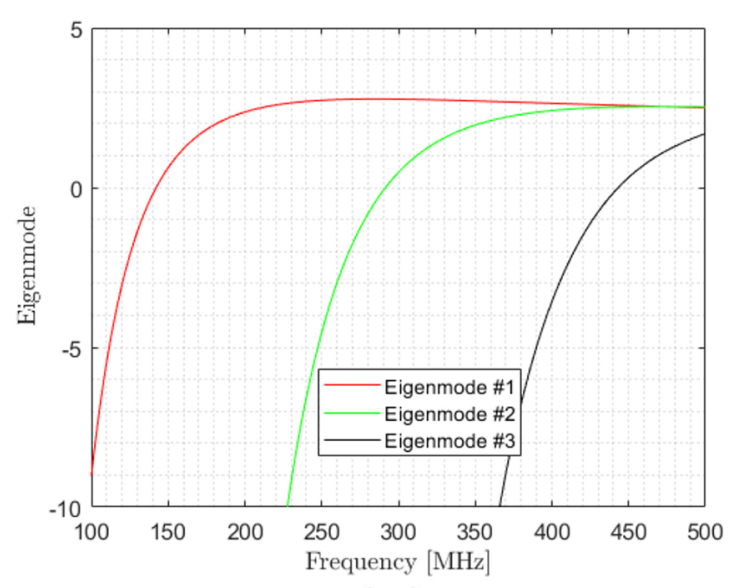

(a)

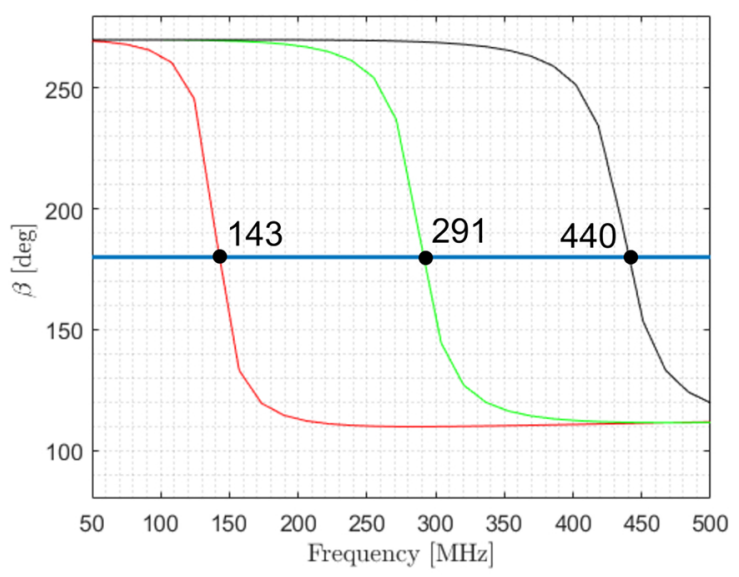

(b)

Figure 1: Three first modes of the metallic strip - (top) eigenmode $\lambda_{n}$ values and (bottom) respective characteristic angles $\beta_{n}$.

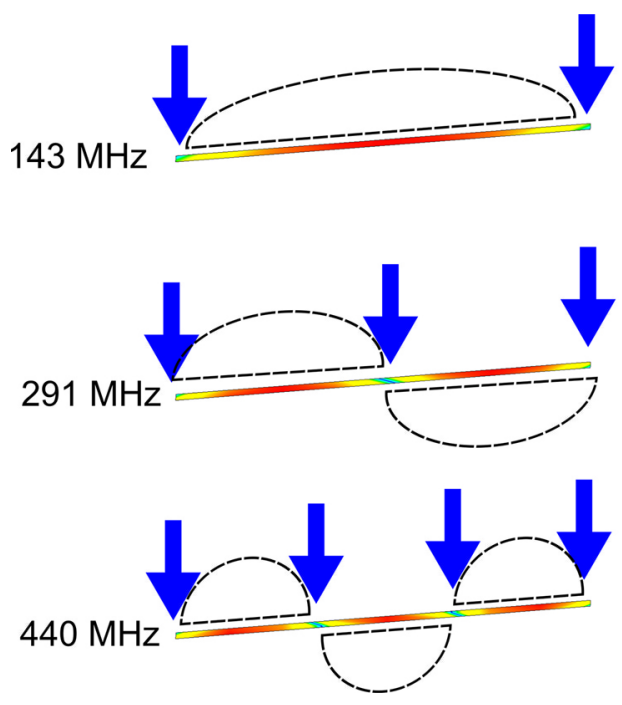

Figure 2: Normalized current surface for the three first modes. 


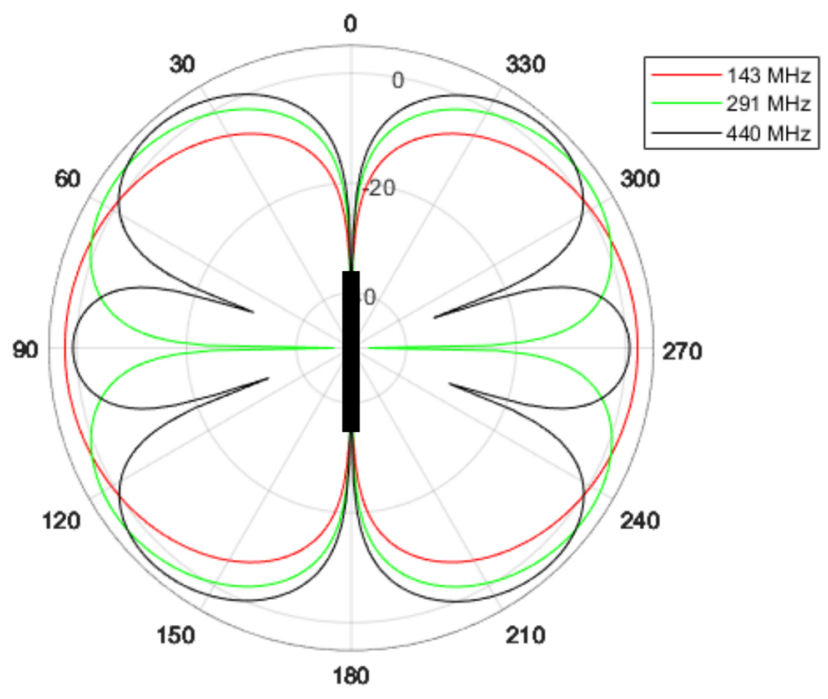

Figure 3: Directivity pattern for the three first modes. The antenna is represented in the dark rectangle in the center of the plot.

available - the so-called inductive couplers are placed on regions where there is a maximum of current density (or a minimum electric field), whereas capacitive couplers are positioned where there is a maximum of electric field (i.e. a minimum current) [8].

\section{Case study II: rectangular patch}

CMA has been used to take advantage of natural modes of resonance of chassis and structures that are not originally employed to radiate. To investigate the modes, a rectangular thin sheet $(10 \mathrm{~cm}$ vs. $5 \mathrm{~cm})$ was simulated. Figure 4 shows the computed first five characteristic angles $\beta_{n}$. Table 2 shows the frequency of the first five modes.

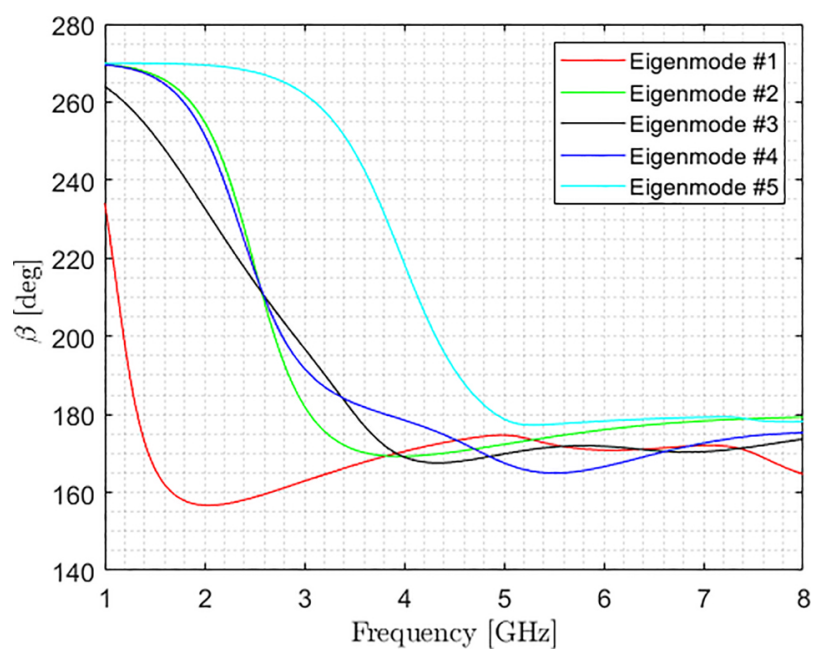

Figure 4: Computed characteristic angles $\beta \mathrm{n}$ of the first five characteristic modes.
Table 2: First five characteristic modes frequencies.

\begin{tabular}{lc}
\hline Mode & Frequency $[\mathrm{GHz}]$ \\
\hline 1 & 1.32 \\
2 & 3 \\
3 & 3.51 \\
4 & 3.83 \\
5 & 4.92 \\
\hline
\end{tabular}

Constrained by the boundary conditions of the structure, the surface current plots of the modes are shown in Figure 5. The shadow gray arrows were placed to emphasize the directions and locations of the maximum currents. As expected, the lowest frequency eigenmode currents are distributed along the larger length side. The other modes fit progressively into the existing structure, according to the boundary conditions.

Upon looking at the position and orientation of the currents it is possible to devise a way to excite a given frequency of interest. The exciter placement and geometry should induce currents in the same direction and shape as the respective mode. The name "exciter" conveys its role, it is not of a radiator - its purpose is actually to
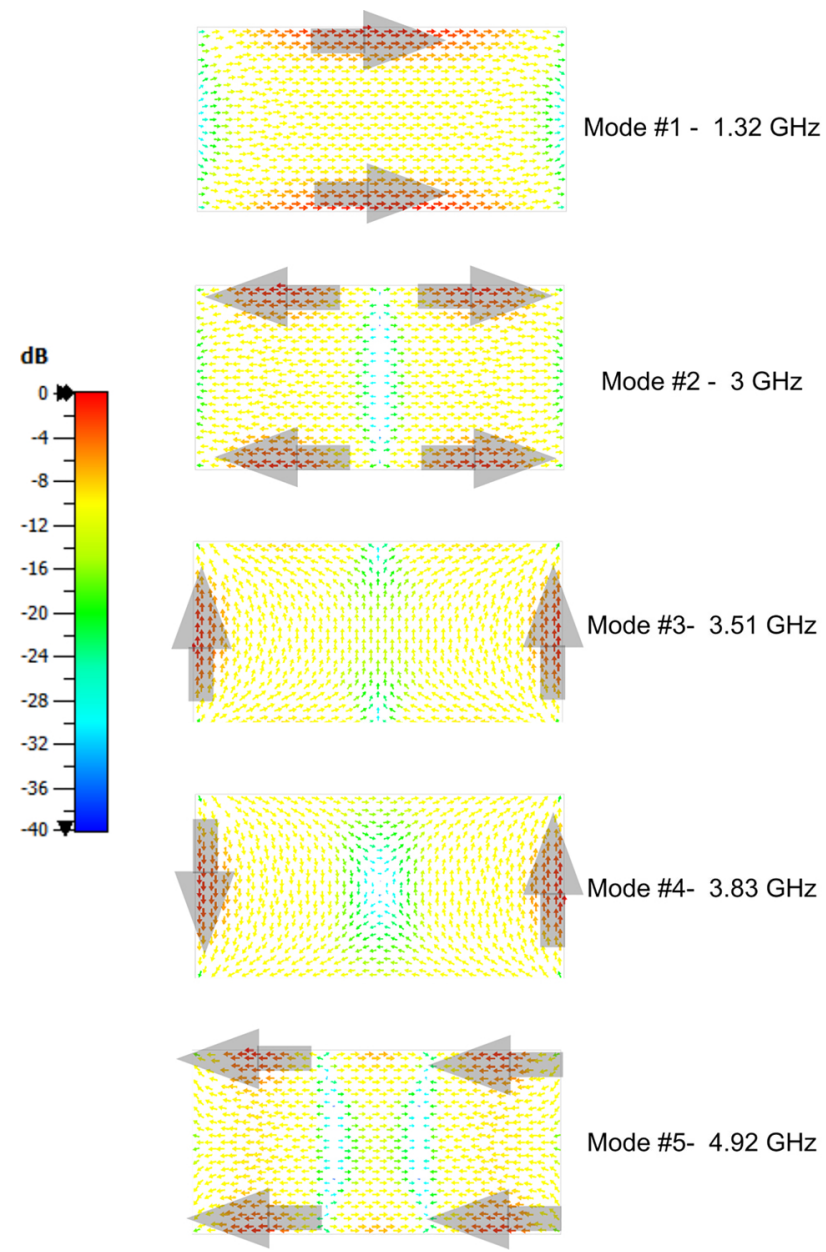

Figure 5: Normalized surface current vectors of the first five characteristic modes. 
couple to the larger structure the electromagnetic energy from the source, which will then fit into a proper mode and radiate the electromagnetic wave.

An example of a real-world object whose shape is rectangular is a mobile phone. The inclusion of antennas to operate in several different frequency bands is a complicated task given the object size. GPS $(1.5 \mathrm{GHz})$, WiFi, Bluetooth and $4 \mathrm{G}(2.5 \mathrm{GHz})$, mobile phone carriers (around $900 \mathrm{MHz}$ and $1800 \mathrm{MHz}$ ) are usually employed in a single phone. Figure 6 shows the computer model of a commercial iPhone $\mathrm{X}$ simulated (dimensions indicated on the figure). For simplicity, the whole case was considered metallic. It can be seen that the computed mode has frequencies exactly on the 900 and $2500 \mathrm{MHz}$-frequency range, which would be useful for covering these services without the need of resonant antennas, using the chassis as a radiator instead, saving up space inside the object.

\section{Case study III: Unmanned Aircraft}

For the case of a UAV (unmanned aerial vehicle), natural modes also arise due to its structure dimensions. The model hereby analyzed is a version of the Israeli Hermes (Figure 7). Vehicles (aircraft, cars, trucks) are natural targets for use of CMA-based antennas, due to their large dimensions and wide use of metallic materials. Given vehicular dimensions, usual modes resonate within the $\mathrm{MHz}$ range (VHF, Very High Frequency). For the sake of simplicity, the whole UAV body was considered to be made of lossless metal, neglecting eventual RAM
(Radiation Absorbent Material) coatings or composite materials used for other intentions. The first three modes of the UAV are computed using the Method of Moments of CST Microwave Studio (Figure 8).

These three modes $(17 \mathrm{MHz}, 21 \mathrm{MHz}$, and $36 \mathrm{MHz})$ are spatially distributed along the object surface according to Figure 9. It shows, on the left, the current density distributions (normalized amplitude). These modes generate farfield patterns (considering the current distribution as a source), respectively shown on the right side of Figure 9. Red arrows were added to show the orientation and placement of the main current distribution, so that one can see that the first mode $(17 \mathrm{MHz})$ generates a dipole-like $3 \mathrm{D}$ gain pattern, whereas the other next mode $(21 \mathrm{MHz})$ has a 90-degree displacement on the farfield and current orientation. The third mode $(36 \mathrm{MHz})$, due to its current distribution with a null right on the fuselage generates a distorted dipole-shaped farfield - besides that the current on each wing has an 180- degrees phase difference. The structure has stabilizers and other additional elements that present non-linear paths to the current, therefore the farfield gain patterns are different from those of ordinary dipoles, which are computed considered the radiators as single straight wires.

From the CMA results, one can grasp the potential bandwidth associated with each mode. A too narrow bandwidth mode might not be be used as antenna since it does not efficiently occupy the available communication channel. According to Figure 8, the first mode has a smaller potential bandwidth, given to its sharp

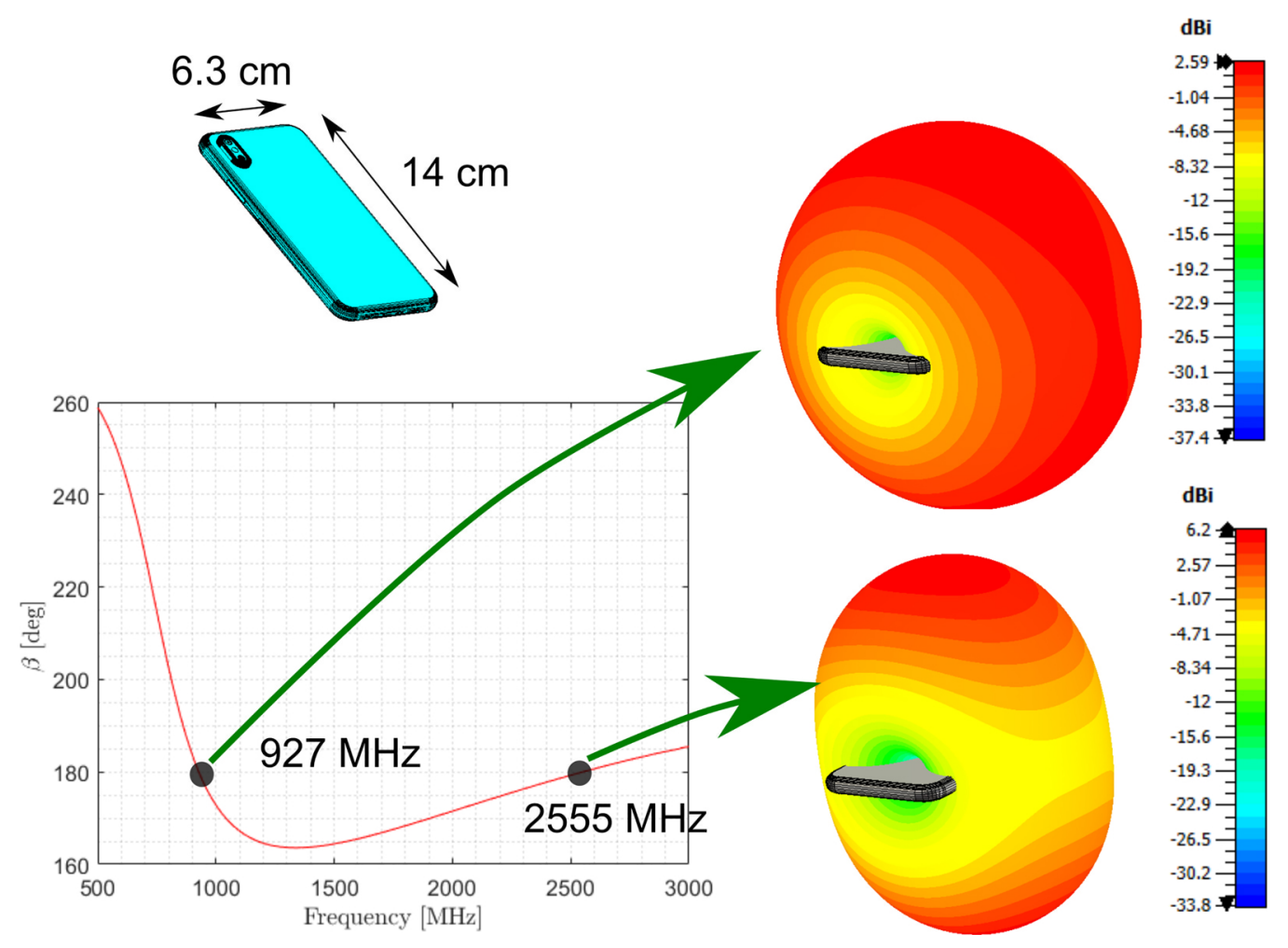

Figure 6: Mobile phone characteristic angle and respective farfield gain patterns. 


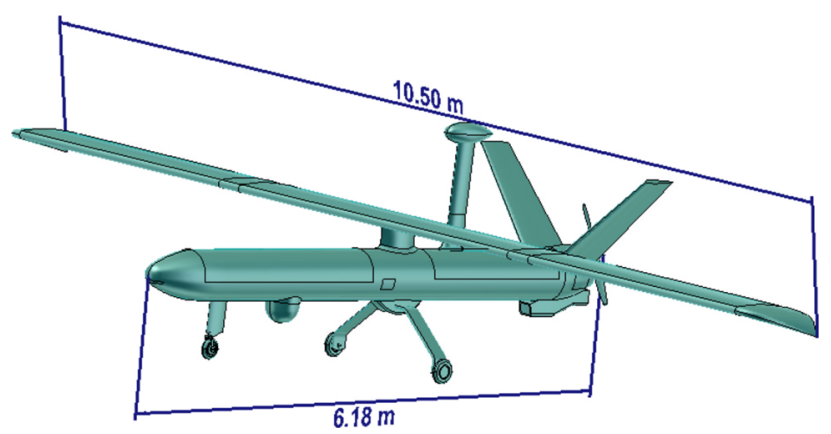

Figure 7: UAV mode with main dimensions - wingspan and fuselage length.

response across the frequency, whereas the third mode has a potential larger bandwidth (smoother response).

From the viewpoint of antenna placement, aircraft represent an involved engineering problem, given the aerodynamic constraints and the influence the metallic structure imposes to the antenna (the electrically large structure couples to the antennas, distorting the fields and detuning the original antenna input impedance). For that specific matter (antennas placed on missiles and jet fighters) patch antennas were invented [7] - the metallic ground plane could shield the radiated fields

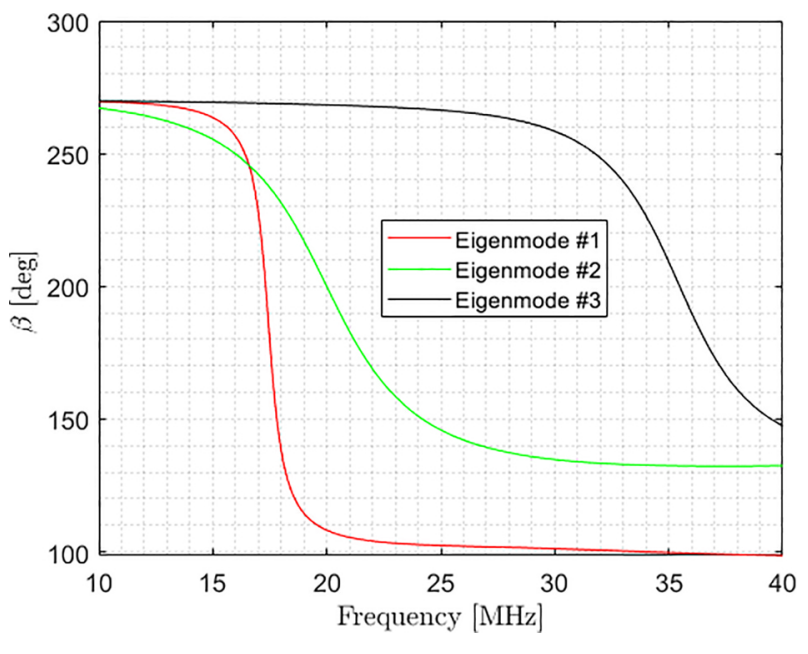

Figure 8: UAV characteristic angles.

from the antenna, and their low profile planar shape can be embedded in a fuselage or wing without much inconvenience. Alternatively, the modes found on CMA can be used so that a small exciter placed on a high amplitude current density region of the UAV will use the fuselage or wing to radiate, therefore there is no need to employ a long (given the $\mathrm{MHz}$ range considered) wire

\section{$17 \mathrm{MHz}$}
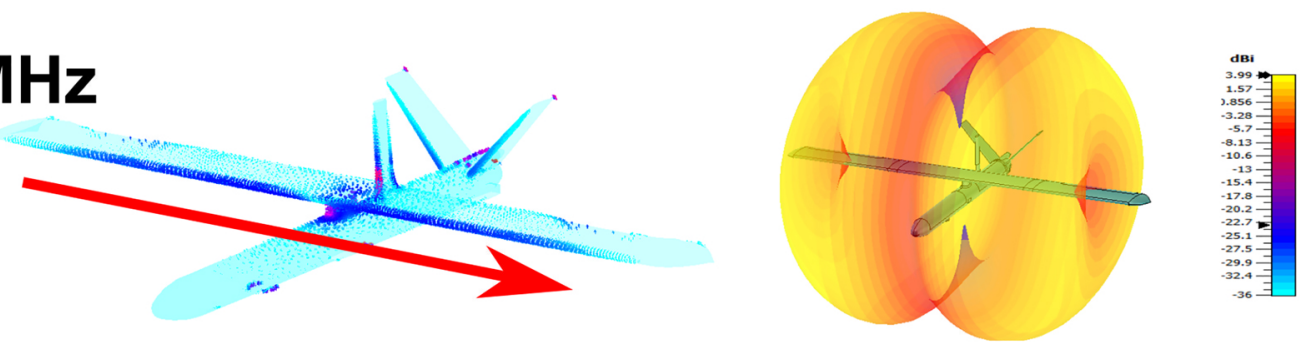

\section{$21 \mathrm{MHz}$}
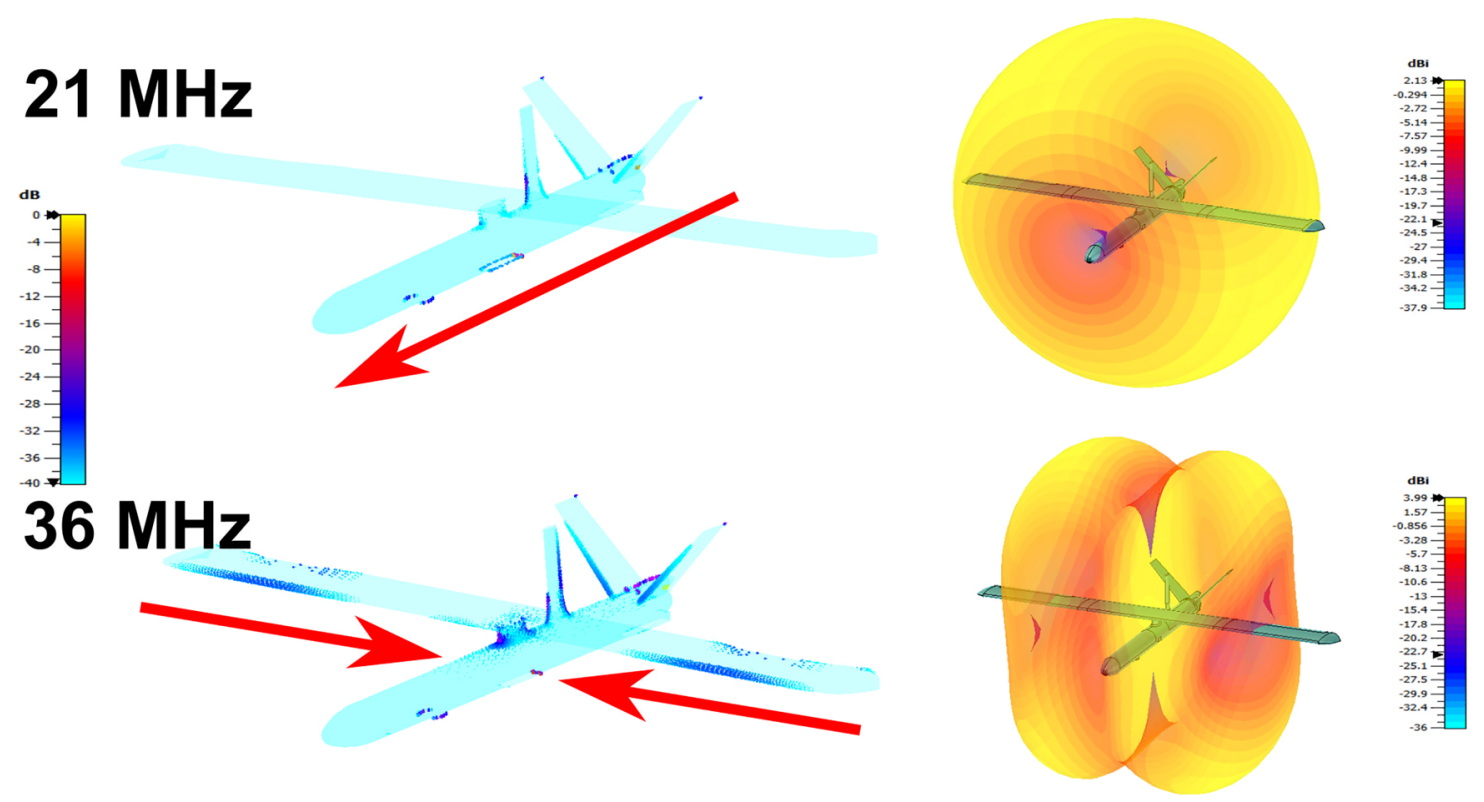

Figure 9: UAV computed normalized surface currents [dB]; left and respective farfield gain patterns; right. 
antenna, which might likely be impractical due to the aerodynamic drag. Besides that, the low gain patterns are nearly omnidirectional, which is attractive in terms of a vehicle that moves away from the control site.

\section{Conclusion}

This article shows applications of the Characteristic Mode Analysis, using a commercial 3D electromagnetic field solver. From the canonical wire antenna up to real-world cases (a mobile phone and an unmanned aerial vehicle), examples are shown where computed results are associated to physical modes. Possible engineering applications are commented based on the insights given by the analyses.

\section{References}

[1] R.J. Garbacz and H. Turpin, IEEE Trans. Antennas Propag. 19, 348 (1971).

[2] R.F. Harrington and J.R. Mautz, IEEE Trans. Antennas Propag. 19, 622 (1971).

[3] M. Cabedo-Fabres and E. Antonino-Daviu, IEEE Trans. Antennas Propag. 49, 52 (2007).

[4] D. Wen, Y. Hao, H. Wang and H. Zhou, IEEE Trans. Antennas Propag. 65, 2671 (2017).

[5] E.M. Santos, C. Molina and A.P.B. Tufaile, Rev. Bras. Ensino Fis. 35, 2507 (2013).

[6] T.R. Hsu, Applied Engineering Analysis (Wiley, New Jersey, 2018).

[7] A.B. Constantine, Antenna Theory Analysis and Design (John Wiley and Sons, New Jersey, 2005).

[8] R. Martens, E. Safin and D. Manteuffel, in LAPC 2011 2011 Loughbrgh. Antennas Propag. Conf. (Loughborough, 2011). 